where $M$ is independent of $n$ and $z$.

There can be developed extensions of the above results to approximation on an arbitrary analytic Jordan curve or on more general point sets bounded by analytic Jordan curves, by rational functions with poles in prescribed points or uniformly distributed on given curves. The present results are intended primarily as illustrations of this general theory.

HARVARD UNIVERSITY AND

ForT RILEy, KaNSAS

\title{
NOTE ON THE COEFFICIENTS OF THE CYCLOTOMIC POLYNOMIAL
}

P. T. BATEMAN

Erdös ${ }^{1}$ has proved that if $A_{n}$ denotes the largest coefficient (in absolute value) of the $n$th cyclotomic polynomial, then for infinitely many $n$

$$
A_{n}>\exp \left\{c_{1}(\log n)^{4 / 3}\right\} .
$$

He also conjectured that a much stronger statement may be true, namely that ${ }^{2}$

$$
A_{n}>\exp \left\{n^{\left(c_{18} / \log \log n\right)}\right\}
$$

holds for some $c_{13}$ and infinitely many $n$, but pointed out that this would be a best result, since

$$
A_{n}<\exp \left\{n^{\left(0_{14} / \log \log n\right)}\right\}
$$

for some $c_{14}$ and all $n$. Erdös suppressed the proof of (B), because his proof was complicated. It is the purpose of this note to give the following short proof of (B).

The cyclotomic polynomial $F_{n}(x)=\prod_{d \mid n}\left(1-x^{d}\right)^{\mu(n / d)}$ is majorized by the power series

$$
\prod_{d \mid n}\left(1+x^{d}+x^{2 d}+\cdots\right) .
$$

Received by the editors September 20, 1948.

${ }^{1}$ Paul Erdös, On the coefficients of the cyclotomic polynomial, Bull. Amer. Math. Soc. vol. 52 (1946) pp. 179-184.

${ }^{2}$ Formulas (A) and (B) were printed incorrectly in Erdös' paper (on the bottom of p. 182). 
Since $F_{n}(x)$ is of degree less than $n$, it is also in fact majorized by the polynomial

$$
\prod_{d \mid n}\left(1+x^{d}+x^{2 d}+\cdots+x^{(n / d-1) d}\right) .
$$

Hence $A_{n}$ is less than the sum of the coefficients of this polynomial. Thus, if $d(n)$ denotes the number of divisors of $n$, we have for sufficiently large $n$

$$
\begin{aligned}
A_{n} & <\prod_{d \mid n}(n / d)=n^{d(n) / 2}=\exp \left\{\frac{1}{2} d(n) \log n\right\} \\
& <\exp \left\{\frac{1}{2} 2^{(1+\epsilon / 2) \log n / \log \log n} \log n\right\} \\
& <\exp \left\{2^{(1+\epsilon) \log n / \log \log n}\right\} \\
& =\exp \left\{n^{(1+\epsilon) \log 2 / \log \log n}\right\},
\end{aligned}
$$

where we have used Wigert's estimation ${ }^{3}$ of $d(n)$. Thus (B) is proved. Added in proof: In a paper to be published in Portugaliae Mathematica, Erdös has given a proof of (A) (for some positive $c_{13}$ and infinitely many $n$ ).

\section{INSTITUTE FOR AdvaNCEd STUdy}

${ }^{3}$ Cf. E. Landau, Handbuch der Lehre von der Verteilung der Primzahlen, pp. 219222, or S. Ramanujan, Collected papers, pp. 44-46. 